\title{
Numerical Computation of Shock Waves in a Spherical Cloud of Cavitation Bubbles
}

\author{
Yi-Chun Wang \\ Assistant Professor, \\ Department of Mechanical Engineering, \\ National Cheng Kung University, Tainan 701, Taiwan \\ Christopher E. Brennen \\ Professor, \\ Division of Engineering and Applied Science \\ California Institute of Technology, Pasadena, California 91125, USA
}

\begin{abstract}
The nonlinear dynamics of a spherical cloud of cavitation bubbles have been simulated numerically in order to learn more about the physical phenomena occurring in cloud cavitation. A finite cloud of nuclei is subject to a decrease in the ambient pressure which causes the cloud to cavitate. A subsequent pressure recovery then causes the cloud to collapse. This is typical of the transient behavior exhibited by a bubble cloud as it passes a body or the blade of a ship propeller. The simulations employ the fully nonlinear continuum mixture equations coupled with the Rayleigh-Plesset equation for the dynamics of bubbles. A Lagrangian integral method is developed to solve this set of equations. It was found that, with strong bubble interaction effects, the collapse of the cloud is accompanied by the formation of an inward propagating bubbly shock wave. A large pressure pulse is produced when this shock passes the bubbles and causes them to collapse. The focusing of the shock at the center of the cloud produces a very large pressure pulse which radiates a substantial impulse to the far field and provides an explanation for the severe noise and damage potential in cloud cavitation.
\end{abstract}

\section{NOMENCLATURE}

A Dimensionless radius of bubble cloud

$A_{0} \quad$ Dimensionless initial cloud radius

$c^{*} \quad$ Sonic speed in bubbly mixture

$C_{P} \quad$ Pressure coefficient

$C_{P \infty} \quad$ Pressure coefficient at infinity from the cloud, $\left(p_{\infty}^{*}-p_{0}^{*}\right) / \frac{1}{2} \rho_{L}^{*} U^{*^{2}}$

$C_{P M I N}$ Minimum pressure coefficient at infinity

$D \quad$ Dimensionless length scale of the low pressure perturbation

$D^{*} \quad$ Length scale of the low pressure perturbation

$f \quad$ Dimensionless frequency

I Dimensionless acoustic impulse

$J \quad$ Jacobian of the transformation from Lagrangian coordinates to Eulerian coordinates

$k \quad$ Polytropic index for the gas inside the bubbles

$p_{a} \quad$ Dimensionless far-field acoustic pressure radiated by the cloud

$p_{\infty}^{*} \quad$ Pressure at infinity from the cloud

$p^{*} \quad$ Fluid pressure

$p_{0}^{*} \quad$ Fluid pressure at undisturbed reference condition

$p_{a}^{*} \quad$ Far-field acoustic pressure radiated by the cloud

$p_{V}^{*} \quad$ Vapor pressure

$r \quad$ Dimensionless Eulerian radial coordinate measured from the center of cloud, $r^{*} / R_{0}^{*}$

$r_{0} \quad$ Dimensionless Lagrangian radial coordinate measured from the center of cloud and equal to $r$ at the undisturbed reference condition

$r^{*} \quad$ Eulerian radial coordinate measured from the center of cloud

$R \quad$ Dimensionless bubble radius

$R_{0} \quad$ Dimensionless initial bubble radius

$R_{0}^{*} \quad$ Initial bubble radius at undisturbed reference condition

Re Reynolds number

$S^{*} \quad$ Surface tension of the liquid

$t \quad$ Dimensionless time

$t_{G} \quad$ Dimensionless duration of the low pressure perturbation

$t^{*} \quad$ Time

$T_{B}^{*} \quad$ Natural period of bubble pulsation 
$T_{C}^{*} \quad$ Time scale of acoustic wave propagation

through the cloud

$u \quad$ Dimensionless fluid velocity

$u^{*} \quad$ Fluid velocity

$U^{*} \quad$ Reference velocity

$V^{*} \quad$ Volume of the cloud

$V_{B M A X}$ Dimensionless maximum total volume of

bubbles in the cloud

$W e \quad$ Weber number

$\alpha \quad$ Void fraction

$\alpha_{0} \quad$ Initial void fraction

$\beta \quad$ cloud interaction parameter, $\alpha_{0}\left(1-\alpha_{0}\right) A_{0}^{2} / R_{0}^{2}$

$\eta \quad$ Dimensionless bubble population per unit

liquid volume, $\eta^{*} R_{0}^{*^{3}}$

$\eta^{*} \quad$ Bubble population per unit liquid volume

$\mu_{E}^{*} \quad$ Effective dynamic viscosity of the liquid

$\omega_{B}^{*} \quad$ Natural frequency of bubble pulsation

$\rho \quad$ Dimensionless mixture density

$\rho_{0} \quad$ Dimensionless mixture density at $t=0$

$\rho_{g} \quad$ Dimensionless gas density inside a bubble, $\rho_{g}^{*} / \rho_{L}^{*}$

$\rho_{g}^{*} \quad$ Gas density inside a bubble

$\rho_{L}^{*} \quad$ Liquid Density

$\sigma \quad$ Cavitation number

$\triangle t \quad$ Time step

\section{INTRODUCTION}

Experimental studies have shown that intensive noise and damage potential are associated with the collapse of a cavitating cloud of bubbles (see, for example, Bark and Berlekom, 1978; Shen and Peterson, 1978, 1980; Bark, 1985; Franc and Michel, 1988; Kubota et al., 1989; Le et al., 1993; Reisman et al., 1994). Moreover, it has been demonstrated that when clouds of cavitation bubbles collapse coherently, they result in greater material damage (see, for example, Soyama et al., 1992) and greater noise generation (see, for example, Reisman and Brennen, 1996) than would be expected from the cumulative effect of the collapse of the individual bubbles which make up the cloud. However, the precise physical phenomena involved in cloud cavitation have not, as yet, been completely identified. This paper presents numerical studies of the nonlinear dynamics of finite clouds of cavitation bubbles. Some preliminary results were presented earlier in Reisman, Wang and Brennen (1997); here we provide more details and further results. The purpose is to explore the mechanisms for the enhanced noise and damage potential associated with cloud cavitation.

Analytical studies of the dynamics of cavitation clouds can be traced to the work of van Wijngaarden (1964) who proposed a continuum model to study the behavior of a collapsing layer of bubbly fluid next to a flat wall and found higher average pressures at the wall as result of the interactive effects of bubbles. Chahine (1982a, 1982b) explored numerical methods which incorporate the individual bubbles using matched asymptotic expansions. Later, d'Agostino and Brennen $(1983,1989)$ investigated the linearized dynamics of a spherical cloud of bubbles using a continuum mixture model coupled with the RayleighPlesset equation for the dynamics of the bubbles. They showed that the interaction between bubbles leads to a coherent dynamics of the cloud, including natural frequencies that can be much smaller than the natural frequencies of individual bubbles. Omta (1987) linearized the Biesheuvel-van Wijngaarden homogeneous flow equations for bubbly mixtures (Biesheuvel and Wijngaarden, 1984) and obtained solutions to the flow in a spherical bubble cloud under a number of simplified assumptions. Thus while the linearized dynamics of clouds of bubbles have been extensively investigated (see also, for example, Prosperetti, 1988; d'Agostino and Brennen, 1983, 1988, 1989), the nonlinear effects in cloud cavitation have received comparatively little attention; in practice, of course, flows with cloud cavitation experience very large pressure perturbations. Both the dynamics of an individual bubble and the bubble/bubble interaction through the hydrodynamics of the surrounding liquid are highly nonlinear. An attempt to understand these nonlinear effects was undertaken in the work of Kumar and Brennen (1991, 1992, 1993). They found weakly nonlinear solutions to a number of cloud problems by retaining only the terms that are quadratic in the amplitude. The nonlinear, chaotic behavior of periodically driven bubble clouds have been recently examined by Smereka and Banerjee (1988) and Birnir and Smereka (1990). These studies employed the methods of dynamical systems analysis and reveal a complicated system of bifurcations and strange attractors in the oscillations of bubble clouds. It is clear that much remains to be learned about the massively nonlinear response of a bubble cloud in a cavitating flow.

All the above researches were based on continuum mixture models which were developed using ensemble volume averaging (Biesheuvel and Wijngaarden, 1984) or time averaging (Ishii, 1975). Effects of bubble dynamics, liquid compressibility and relative motion between phases can be included. Such continuum mixture models have been successfully applied to investigate shock wave propagation in liquids containing small gas bubbles (see, for example, Noordzij, 1973; Noordzij and van Wijngaarden, 1974; Kameda and Matsumoto, 1995).

Another approach to the modeling of the interaction dynamics of cavities was developed by Chahine and his coworkers (Chahine and Duraiswami, 1992; Chahine et al., 1992). Three-dimensional boundary element methods 
have been employed to simulate the deformations of the individual bubbles within collapsing clouds in inhomogeneous flow fields or close to solid boundaries. However, most clouds contain many thousands of bubbles, impossible to handle by any high speed computer at this time. It therefore is advantageous to examine the nonlinear behavior of cavitation clouds using continuum mixture models. The recent numerical modeling of unsteady cavitating flows on a two-dimensional hydrofoil by Kubota et al. (1992) is an important step in this direction. Solving the Navier-Stokes equations of the mixture coupled with the Rayleigh equation for bubble dynamics, they reproduced the shedding of cavitation clouds and the generation of vortex cavitation. Unfortunately, there appear to be some limitations in their approach. They artificially prevented the bubbles from collapsing to a size smaller than the initial value. Under these circumstances, the collapse of the bubbles is severely suppressed and highly nonlinear phenomena such as the formation of shock waves are eliminated. Parenthetically, it is shown in this paper that very large pressure pulses can occur due to the violent bubble collapse. In Wang (1996), it was shown that such large pulses cause substantial computational difficulties.

Another perspective on the subject of collapsing clouds was that introduced by Mørch, Hanson and Kedrinskii (Mørch, 1980, 1981, 1982; Hanson et al., 1981). They speculated that the collapse of a cloud of bubbles involves the formation and inward propagation of a shock wave and that the geometric focusing of this shock at the center of a cloud creates the enhancement of the noise and damage potential associated with cloud collapse. However, they assumed that the bubbles are completely annihilated after the shock passing. Fully nonlinear solutions for spherical cloud dynamics were first obtained by Wang and Brennen (1994, 1995a, 1995b). Their computational results show that the continuum models of the cloud indeed manifest the shock wave phenomena and thus confirm the idea put forward by Mørch, Hanson et al. Recently, Reisman et al. (1997) measured very large impulsive pressures on the suction surface of an oscillating hydrofoil experiencing cloud cavitation. They demonstrate that these pressure pulses are associated with the propagation of bubbly shock waves.

\section{BASIC EQUATIONS}

Consider a spherical bubble cloud surrounded by an unbounded pure liquid as shown in figure 1. The liquid is at rest infinitely far from the cloud. Compared to the large compressibility of the cloud, the pure liquid is assumed incompressible. It is assumed that the population of bubbles per unit volume of liquid, $\eta$, within the cloud, is piecewise

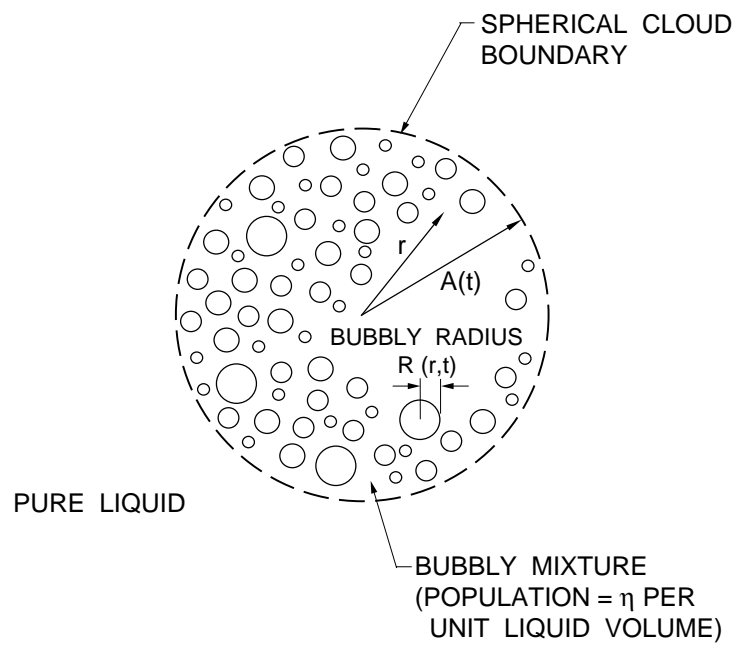

Figure 1: Schematic of a spherical cloud of bubbles.

uniform initially and that there is no coalescence or breakup of bubbles. Since relative motion between the phases is neglected and the mass of liquid vaporized or condensed is also neglected, it follows that $\eta$ remains both constant and piecewise uniform within the cloud. The radius of the cloud is represented by $A(t)$, a function of time $t$. The bubble radius in the cloud is $R(r, t)$, a function of radial coordinate $r$ and time. The bubbles are assumed to be spherical and to contain uniform liquid vapor and residual permanent gas. The problem to be solved is as follows. The cloud and the whole domain of liquid are initially in equilibrium. Starting at $t=0$, a pressure perturbation, $C_{P \infty}(t)$, is imposed on the pure liquid at infinity and the response of the cloud to this pressure perturbation is sought.

The variables mentioned above and in all the following figures and equations are non-dimensionalized using the initial bubble radius, $R_{0}^{*}$, and a reference flow velocity, $U^{*}$. All quantities with superscript * represent dimensional values; without this superscript the quantities are nondimensional. Thus, the non-dimensional bubble radius is $R=R^{*} / R_{0}^{*}$, the non-dimensional bubble population per unit liquid volume is $\eta=\eta^{*} R_{0}^{*^{3}}$, the non-dimensional radial coordinate is $r=r^{*} / R_{0}^{*}$, and the non-dimensional time is $t=t^{*} U^{*} / R_{0}^{*}$.

The basic equations used are those of d'Agostino and Brennen (1983, 1988, 1989) except that all the nonlinear convective terms are retained since these are important in the context of the highly nonlinear growth and collapse of the cloud. The dimensionless forms of the continuity and momentum equations for the spherical bubbly flow are:

$$
\frac{1}{r^{2}} \frac{\partial\left(r^{2} u\right)}{\partial r}=\frac{12 \pi \eta R^{2}}{3+4 \pi \eta R^{3}} \frac{D R}{D t} \quad ; \quad r \leq A(t)
$$




$$
\frac{D u}{D t}=-\frac{1}{6}\left(3+4 \pi \eta R^{3}\right) \frac{\partial C_{P}}{\partial r} \quad ; \quad r \leq A(t)
$$

where $D / D t \equiv \partial / \partial t+u \partial / \partial r$ is the Lagrangian derivative, $u(r, t)$ is the mixture velocity, $C_{P}(r, t)=\left(p^{*}(r, t)-\right.$ $\left.p_{0}^{*}\right) / \frac{1}{2} \rho_{L}^{*} U^{*^{2}}$ is the mixture pressure coefficient, $p^{*}(r, t)$ is the mixture pressure, $p_{0}^{*}$ is the initial equilibrium pressure, and $\rho_{L}^{*}$ is the liquid density. The bubble population per unit liquid volume, $\eta$, is related to the void fraction, $\alpha$, by $\frac{4}{3} \pi R^{3} \eta=\alpha /(1-\alpha)$. The interactions of the bubbles with the flow are modeled by the Rayleigh-Plesset equation (Knapp et al., 1970; Plesset and Prosperetti, 1977) which connects the local mixture pressure coefficient, $C_{P}$, to the bubble radius, $R$ :

$$
\begin{aligned}
R \frac{D^{2} R}{D t^{2}} & +\frac{3}{2}\left(\frac{D R}{D t}\right)^{2}+\frac{\sigma}{2}\left(1-R^{-3 k}\right)+\frac{4}{R e} \frac{1}{R} \frac{D R}{D t} \\
& +\frac{2}{W e}\left(R^{-1}-R^{-3 k}\right)+\frac{1}{2} C_{P}=0
\end{aligned}
$$

where $\sigma=\left(p_{0}^{*}-p_{V}^{*}\right) / \frac{1}{2} \rho_{L}^{*} U^{*^{2}}$ is the cavitation number and $p_{V}^{*}$ is the partial pressure of vapor inside the bubble. The partial pressure of non-condensable gas (it is assumed the mass of gas is constant) does not appear explicitly in (3) because the initial equilibrium condition has been employed to eliminate this quantity. It has been assumed that the non-condensable gas inside the bubbles behaves polytropically with an index $k$. From the definition of cavitation number, $\sigma$, we know that small values of $\sigma$ imply that the initial equilibrium pressure is close to the vapor pressure and the bubbles therefore cavitate more readily. We define a Reynolds number, $R e=\rho_{L}^{*} U^{*} R_{0}^{*} / \mu_{E}^{*}$, where $\mu_{E}^{*}$ is the effective viscosity of liquid which incorporates the various bubble-damping mechanisms, namely acoustic, thermal, and viscous damping, described by Chapman and Plesset (1971), We also define a Weber number, $W e=\rho_{L}^{*} U^{*^{2}} R_{0}^{*} / S^{*}$, where $S^{*}$ is the surface tension of the liquid.

The Rayleigh-Plesset equation (3) neglects the local pressure perturbations experienced by the individual bubble due to the growth or collapse of its neighbor. d'Agostino and Brennen (1989), Nigmatulin (1991) and Sangani (1991) have shown that, for a mixture with randomly distributing bubbles, the correction factors for local pressure perturbations are of order of the void fraction, $\alpha$, or higher. While a second-order correction could be incorporated without much difficulty, the void fractions considered in the present work are only few percent so that these higher order effects are neglected.

The boundary condition on the surface of the cloud, $r=$ $A(t)$, is obtained as follows. The spherically symmetric incompressible liquid flow outside the cloud, $r \geq A(t)$, must have a solution of the form:

$$
\begin{gathered}
u(r, t)=\frac{C(t)}{r^{2}} ; r \geq A(t) \\
C_{P}(r, t)=C_{P \infty}(t)+\frac{2}{r} \frac{d C(t)}{d t}-\frac{C^{2}(t)}{r^{4}} ; r \geq A(t)
\end{gathered}
$$

where $C(t)$ is an integration constant to be determined and $C_{P \infty}(t)$ is the imposed pressure perturbation coefficient at infinity which will be described later. By substituting the values of $u$ and $r$ at the boundary of the cloud in (4), $C(t)$ can be determined as

$$
C(t)=A^{2}(t) u(A(t), t)
$$

Substituting (6) and $r=A(t)$ into (5), we obtain the time-dependent boundary condition at the surface of the cloud:

$$
\begin{aligned}
C_{P}(A(t), t)= & C_{P \infty}(t)+\frac{2}{A(t)} \frac{d\left[A^{2}(t) u(A(t), t)\right]}{d t} \\
& -u^{2}(A(t), t)
\end{aligned}
$$

At the center of cloud, the symmetry of the problem requires

$$
u(0, t)=0
$$

In the context of cavitating flows it is appropriate to assume that, at time $t \leq 0$, the whole flow field is in equilibrium. It is also assumed, for simplicity, that all the bubbles have the same initial size. Therefore, the following initial conditions should be applied:

$$
R(r, 0)=1, \frac{D R}{D t}(r, 0)=0, u(r, 0)=0, C_{P}(r, 0)=0
$$

The mathematical model of equations (1), (2), and (3) is complete and, after applying appropriate initial and boundary conditions, can, in theory, be solved to find the unknowns $C_{P}(r, t), u(r, t)$, and $R(r, t)$ for any bubbly cavitating flow with spherical symmetry. However, the nonlinearities in the Rayleigh-Plesset equation and in the Lagrangian derivative, $D / D t$, present considerable computational impediments.

In (7), $C_{P \infty}(t)=\left(p_{\infty}^{*}(t)-p_{0}^{*}\right) / \frac{1}{2} \rho_{L}^{*} U^{*^{2}}$ and $p_{\infty}^{*}(t)$ is the far-field pressure perturbation experienced by the cloud in a cavitating flow. For the purposes of the present calculations, a simple sinusoidal form is chosen for $C_{P \infty}(t)$ since previous investigations have shown that the results are not very sensitive to the precise functional form of $C_{P \infty}(t)$ :

$C_{P \infty}(t)= \begin{cases}\frac{1}{2} C_{P M I N}\left[1-\cos \left(2 \pi t / t_{G}\right)\right] & 0<t<t_{G} \\ 0 & ; t<0 \\ & \text { and } t>t_{G}\end{cases}$ 
where $C_{P M I N}$ is the minimum pressure coefficient imposed on the cloud and $t_{G}$ is the non-dimensional duration of the pressure perturbation. Consequently, for a cloud flowing with velocity $U^{*}$ past a body of size $D^{*}$, the order of magnitude of $t_{G}$ will be $D^{*} / R_{0}^{*}$, and $C_{P M I N}$ will be the minimum pressure coefficient of the flow.

\section{NUMERICAL METHOD}

The natural framework for the present problem is a Lagrangian coordinate system based on the mixture velocity, in which all the nonlinear convective terms in the mixture conservation equations, (1), (2), and RayleighPlesset equation, (3), are eliminated. A Lagrangian integral method based on the integral representation of the continuity and momentum equations in the Lagrangian coordinates, $\left(r_{0}, t\right)$, has been developed, in which $r_{0}$ is the radial distance from the center of the cloud at initial time $t=0$. The values of other quantities at $t=0$ are also denoted by a subscript 0 . Therefore, the density of a mixture material element, $\rho\left(r_{0}, t\right)$, is related to its initial density, $\rho_{0}\left(r_{0}\right)$, by

$$
\frac{\rho\left(r_{0}, t\right)}{\rho_{0}\left(r_{0}\right)}=\frac{1}{J}
$$

where $J$ is the Jacobian of the coordinate transformation from Lagrangian to Eulerian coordinates: $r_{0} \longmapsto r\left(r_{0}, t\right)$, and has the following expression in the spherically symmetric configuration:

$$
J=\frac{r^{2}}{r_{0}^{2}} \frac{\partial r}{\partial r_{0}}
$$

This also represents the ratio of the current material volume to its initial volume.

The position of a mixture particle can be obtained by integrating (11).

$$
r\left(r_{0}, t\right)=\left\{\frac{3}{3+4 \pi \eta} \int_{0}^{r_{0}} \xi^{2}\left[3+4 \pi \eta R^{3}(\xi, t)\right] d \xi\right\}^{1 / 3}
$$

where $\xi$ is a dummy integration variable and we approximate $\rho\left(r_{0}, t\right) / \rho_{0}\left(r_{0}\right) \approx\left[1-\alpha\left(r_{0}, t\right)\right] /\left[1-\alpha_{0}\left(r_{0}\right)\right]$ since the liquid density is very much larger than the vapor density. Note that the boundary condition $r\left(r_{0}, t\right)=0$ at the center of the cloud has been used to eliminate an integration constant. The mixture velocity can be obtained by differentiating (13):

$$
\begin{aligned}
u\left(r_{0}, t\right) & =\frac{\partial r\left(r_{0}, t\right)}{\partial t} \\
& =\frac{12 \pi \eta}{(3+4 \pi \eta) r^{2}\left(r_{0}, t\right)} \int_{0}^{r_{0}} \frac{\partial R(\xi, t)}{\partial t} R^{2}(\xi, t) \xi^{2} d \xi
\end{aligned}
$$

The mixture momentum equation, (2), and the boundary condition at the surface of the cloud, (7), have the following forms in the Lagrangian coordinate framework:

$$
\begin{aligned}
\frac{\partial C_{P}\left(r_{0}, t\right)}{\partial r_{0}} & =-\frac{6}{3+4 \pi \eta R^{3}\left(r_{0}, t\right)} \frac{\partial u\left(r_{0}, t\right)}{\partial t} \frac{\partial r\left(r_{0}, t\right)}{\partial r_{0}} \\
C_{P}\left(A_{0}, t\right)= & C_{P \infty}(t)+\frac{2}{r\left(A_{0}, t\right)} \frac{d}{d t}\left[r^{2}\left(A_{0}, t\right) u\left(A_{0}, t\right)\right] \\
& -u^{2}\left(A_{0}, t\right)
\end{aligned}
$$

After substituting (13) and (14) in the right-hand side of (15) and integrating from $r_{0}$ to $A_{0}$ using the boundary condition (16), an integral equation for mixture pressure coefficient, $C_{P}\left(r_{0}, t\right)$, is found:

$$
\begin{aligned}
C_{P}\left(r_{0}, t\right)= & \frac{6}{3+4 \pi \eta}\left[\int_{r_{0}}^{A_{0}} \frac{g\left(\xi, t ; C_{P}\right)-2 r(\xi, t) u^{2}(\xi, t)}{r^{4}(\xi, t)} \xi^{2} d \xi\right] \\
& +\frac{2 g\left(A_{0}, t\right)}{r\left(A_{0}, t\right)}-u^{2}\left(A_{0}, t\right)+C_{P \infty}(t)
\end{aligned}
$$

where

$$
\begin{aligned}
g\left(\xi, t ; C_{P}\right)= & \frac{12 \pi \eta}{3+4 \pi \eta} \int_{0}^{\xi}\left\{\frac{2}{W e}\left[R^{1-3 k}(\zeta, t)-1\right]\right. \\
& -\frac{4}{R e} \frac{\partial R(\zeta, t)}{\partial t}+\frac{\sigma}{2} R(\zeta, t)\left[R^{-3 k}(\zeta, t)-1\right] \\
& +\frac{1}{2} R(\zeta, t)\left[\left(\frac{\partial R(\zeta, t)}{\partial t}\right)^{2}-C_{P}((\zeta, t)]\right\} \zeta^{2} d \zeta
\end{aligned}
$$

Here the Rayleigh-Plesset equation, (3), has been used to eliminate the bubble wall acceleration, $\partial^{2} R\left(r_{0}, t\right) / \partial t^{2}$.

These equations form the basis of the present method for solving for these flows. More explicitly, a complete integration time step proceeds as follows:

1. At each Lagrangian node, $r_{0}, R\left(r_{0}, t+\Delta t\right)$ and $\partial R\left(r_{0}, t+\triangle t\right) / \partial t$ are calculated using an explicit time marching scheme (a Runge-Kutta scheme) based on the known solution at the previous time step, $R\left(r_{0}, t\right)$, $\partial R\left(r_{0}, t\right) / \partial t$ and $\partial^{2} R\left(r_{0}, t\right) / \partial t^{2}$.

2. With $R\left(r_{0}, t+\triangle t\right)$ and $\partial R\left(r_{0}, t+\triangle t\right) / \partial t$, equations (13) and (14) can be integrated to obtain $r\left(r_{0}, t+\triangle t\right)$ and $u\left(r_{0}, t+\triangle t\right)$

3. With the results of steps 1 and 2 , we can iterate upon equation (17) to find $C_{P}\left(r_{0}, t+\triangle t\right)$. Then the Rayleigh-Plesset equation (3) can be used to find $\partial^{2} R\left(r_{0}, t+\triangle t\right) / \partial t^{2}$. Under-relaxation was necessary to make this iterative process converge. This and other numerical difficulties are discussed in Wang (1996). 


\section{Proceed to next time step.}

The interval of each time step is automatically adjusted during the computation to ensure that the maximum fractional change of bubble radius in the cloud between any two consecutive times does not exceed some specific value (typically, 5\%). This is essential for time marching through a violent bubble collapse. The number of Lagrangian nodes is 100 in all the computational results presented here. Nodal numbers of 200 and 400 were used to check the grid independence of the results.

\section{RESULTS AND DISCUSSION}

The following typical flow variables were chosen to illustrate the calculated results. A cloud of nuclei, composed of air bubbles of initial radius $R_{0}^{*}=100 \mu \mathrm{m}$ in water at $20^{\circ} \mathrm{C}\left(\rho_{L}^{*}=1000 \mathrm{~kg} / \mathrm{m}^{3}, \mu_{L}^{*}=0.001 \mathrm{~N} \mathrm{~s} / \mathrm{m}^{2}\right.$, $S^{*}=0.0728 \mathrm{~N} / \mathrm{m}$ ) flows with velocity $U^{*}=10 \mathrm{~m} / \mathrm{s}$ through a region of low pressure characterized by equation (10). The computation is performed for different combinations of the following parameters: the minimum pressure coefficient, $C_{P M I N}$, of -0.7 and -0.75 ; a non-dimensional duration of the pressure perturbation, $t_{G}$, ranging from 50 to 1000 ; an initial void fraction, $\alpha_{0}$, ranging from $0.02 \%$ to $5 \%$; a cavitation number, $\sigma$, ranging from 0.4 to 0.65 ; a non-dimensional cloud radius, $A_{0}$, of 32,100 , and 312 . These ranges of values of $A_{0}$ and $t_{G}$ correspond to the ratio of the length-scale of the pressure perturbation to the initial radius of the cloud, $D / A_{0}$, of 0.5 to 31.25 , values which cover the range of experimental observations. The Reynolds number, based on the reference flow velocity, initial bubble radius, the liquid density, and the effective viscosity, is 28.6 in all the cases presented. Recall that an effective liquid viscosity, $\mu_{E}^{*}=0.035 \mathrm{~N} \mathrm{~s} / \mathrm{m}^{2}$, is used in place of actual liquid viscosity to incorporate the various bubble damping mechanisms (Chapman and Plesset 1971).

\subsection{Cloud Interaction Parameter}

One of the complexities of multiphase flows is the existence of different characteristic time or length scales and the interactions between them. In cloud cavitation one of the most important factors is the effect of the bubble dynamics on the global flow fields. The parameter controlling this effect is $\beta \equiv \alpha_{0}\left(1-\alpha_{0}\right) A_{0}^{2} / R_{0}^{2}$, which will be termed the "cloud interaction parameter." Here, $\alpha_{0}$ is the initial void fraction of the cloud, $A_{0}$ is the initial cloud radius and $R_{0}$ is the initial bubble radius. In the present study, we will show that the nonlinear cloud dynamics were strongly dependent on this parameter. Earlier linear and weakly nonlinear studies of cloud dynamics
(d'Agostino and Brennen, 1983, 1989; Kumar and Brennen, 1991, 1992, 1993) showed that the cloud natural frequency is strongly dependent on this parameter. If $\beta$ is small, the natural frequency of the cloud is close to that of the individual bubbles in the cloud. In other words, the bubbles in the cloud tend to behave as individual units in an infinite fluid and the bubble/bubble interaction effects are minor. Then the dynamic effects of the cloud are approximately the sum of the effects of the individual bubbles in the cloud. On the other hand the bubble interaction effects in the cloud are dominant when the value of $\beta$ is greater than order one. Then the collective oscillation of bubbles in the cloud results in a cloud natural frequency which is lower than the natural frequency of individual bubbles.

In the expression of $\beta, A_{0}$ is the macroscopic lengthscale in the flow, $R_{0}$ characterizes the microscale, and $\alpha_{0}\left(1-\alpha_{0}\right) \approx \alpha_{0}$ represents the concentration of the dispersed phase. It is useful to mention one physical interpretation of $\beta$. The sonic speed in a bubbly mixture of void fraction $\alpha_{0}$ without viscosity and surface tension effects is approximately (see, for example, Brennen 1995)

$$
c^{*}=\left[\frac{k p^{*}}{\alpha_{0}\left(1-\alpha_{0}\right) \rho_{L}^{*}}\right]^{1 / 2}
$$

where $p^{*}$ is the mixture pressure and $k$ is the polytropic index of the gas inside the bubble. The natural frequency of bubbles in the mixture is approximately

$$
\omega_{B}^{*}=\left(\frac{3 k p^{*}}{\rho_{L}^{*} R_{0}^{*}}\right)^{1 / 2}
$$

where $R_{0}^{*}$ is the bubble radius. But the characteristic global dimension of the flow is the radius of the cloud, $A_{0}^{*}$, and, consequently, there are two dynamic time-scales in the flow: the time-scale of bubble dynamics, $T_{B}^{*} \equiv 1 / \omega_{B}^{*}$, and the time-scale of wave propagation through the cloud, $T_{C}^{*} \equiv 1 / \omega_{C}^{*}=A_{0}^{*} / c^{*}$. The ratio of these two characteristic times is

$$
\frac{T_{C}^{*}}{T_{B}^{*}} \approx\left[\frac{\alpha_{0}\left(1-\alpha_{0}\right) A_{0}^{*^{2}}}{R_{0}^{*^{2}}}\right]^{1 / 2}=\sqrt{\beta}
$$

Therefore, $\beta$ determines the ratio of the two characteristic times in the flow. If $\beta$ is small, the bubbles are little affected by the global perturbations in the flow and the global interactions are weak. On the other hand, if $\beta$ is much larger than order one, the bubble dynamics can effectively influence the large scale perturbations and contribute to the global dynamics of the cloud. 


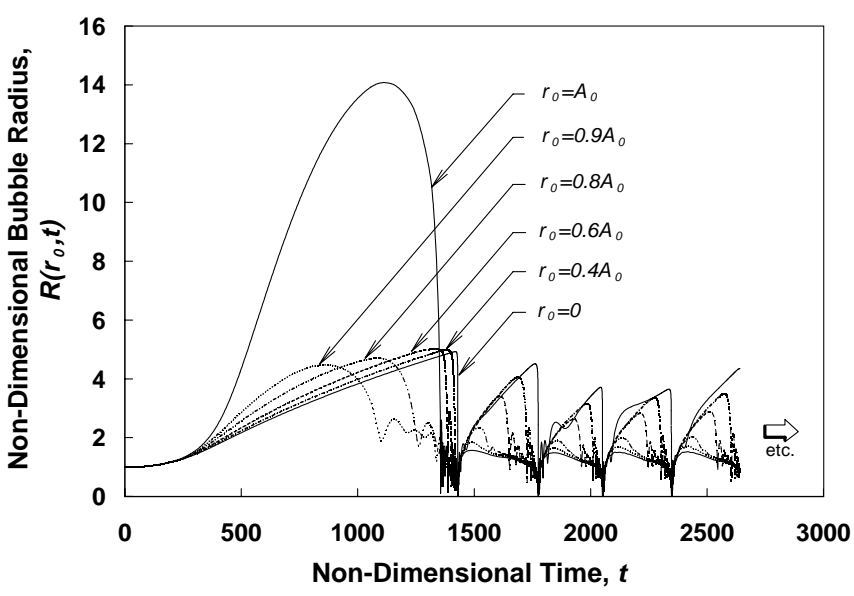

Figure 2: The time history of the dimensionless bubble size at six different Lagrangian positions in the cloud for $\sigma=0.4, C_{P M I N}=-0.7, \alpha_{0}=5 \%, A_{0}=100$, and a ratio of the low pressure perturbation length-scale to initial cloud radius, $D / A_{0}=10$ (corresponds to $t_{G}=1000$ ). The cloud interaction parameter, $\beta$, is 475 in this case.

\subsection{Nonlinear Growth and Collapse of the Cloud with Various Cloud Interaction Parameters, $\beta$}

For the case of a large value of $\beta$, figure $2(\beta=475)$ presents typical time histories of bubble radius for six different Lagrangian locations, $r_{0}$, within the cloud, from the surface, $r_{0}=A_{0}$, to the center, $r_{0}=0$. It is clear that the bubble growth rate decreases greatly with increasing distance from the cloud surface. This is due to bubble/bubble interaction in the cloud. The bubbles in the interior are shielded by the surface shell of bubbles and grow to a smaller maximum size. This shielding effect is typical of the bubble interaction phenomenon appearing in the earlier investigations of cloud dynamics (see, for example, d'Agostino and Brennen, 1983, 1989; Omta, 1987; Smereka and Banerjee, 1988; Chahine and Duraiswami, 1992).

After the recovery of the ambient pressure, bubbles near the surface (at $r_{0}=0.9 A_{0}$ in the present case) of the cloud start to collapse first and the collapse propagates inward. Meanwhile, bubbles on the surface of the cloud keep growing due to their large growth rate and collapse later. If the duration of the pressure perturbation, $t_{G}$, is sufficiently short (say, $t_{G}=500$ ), the collapse is forced to start from the surface of the cloud (see Wang and Brennen, 1995b). Note that the shielding effect causes the bubbles in the interior region to continue to grow even after the surface-layer bubbles have collapsed.

As a result of the inward propagating collapse, a bubbly shock wave develops. To illustrate a typical transient

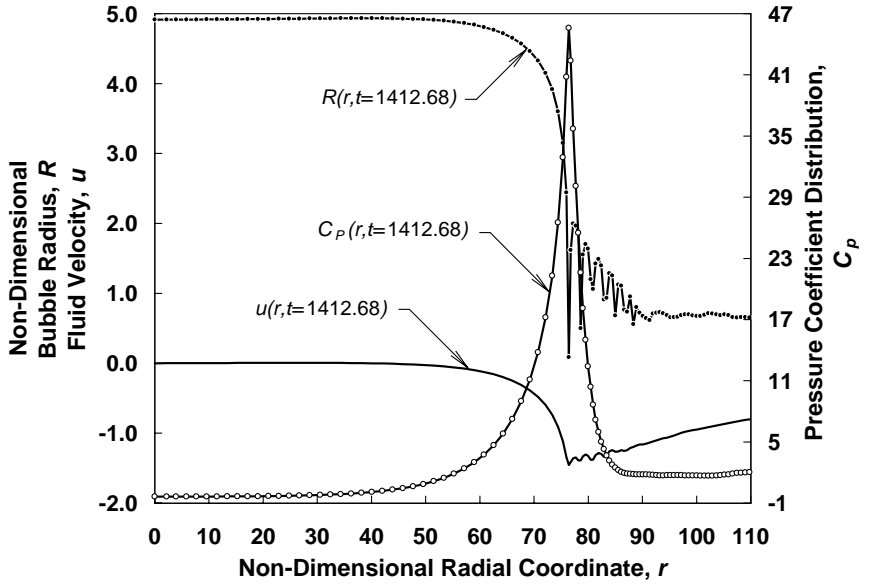

Figure 3: Bubble size, fluid velocity, and pressure distributions in the inward propagating shock wave at a sample moment in time, $t=1412.68$. Parameters as in figure 2 .

structure of this shock wave, figure 3 shows spatial distribution of bubble radius, fluid velocity, and fluid pressure coefficient at one moment in time. The shock wave has progressed inward to a Eulerian position, $r \approx 76$. The wave front of the shock can be easily identified by the bubble collapse front. Unlike gas dynamic shock waves, the bubbly shock has an oscillatory structure behind the shock front which involves a series of rebounds and secondary collapses. This structure is very similar to that of the gas/liquid shocks investigated by Noordzij and van Wijngaarden (1974) (see also Brennen, 1995, §6.9). The locations with small bubble size represent regions of low void fraction and higher pressure due to the local bubble collapse. It is clear that the collapse of bubbles induces inward flow acceleration which creates a local pressure gradient and promotes more violent collapse of the neighboring bubbles. As the shock front passes bubbles and causes them to collapse, a very large pressure pulse can be produced, as shown in figure 3 . The dimensional magnitude of the peak is about $46 \mathrm{~atm}$ in this case. Oscillations in the pressure resulting from secondary collapses do occur but are dwarfed by the primary pressure pulse and are not therefore seen in figure 3 .

The shock wave strengthens considerably as it propagates into the cloud primarily because of the focusing effect of the spherical configuration. The strengthening of the shock can be seen in figure 2; the closer the bubbles are to the cloud center, the smaller the size to which they collapse. Very complicated bubble-bubble interactions are observed when the focusing shock reaches the center of the cloud (at $t \approx 1430$ in the case shown). Very high pressures are generated which cause a rebound of the cloud. Then a 


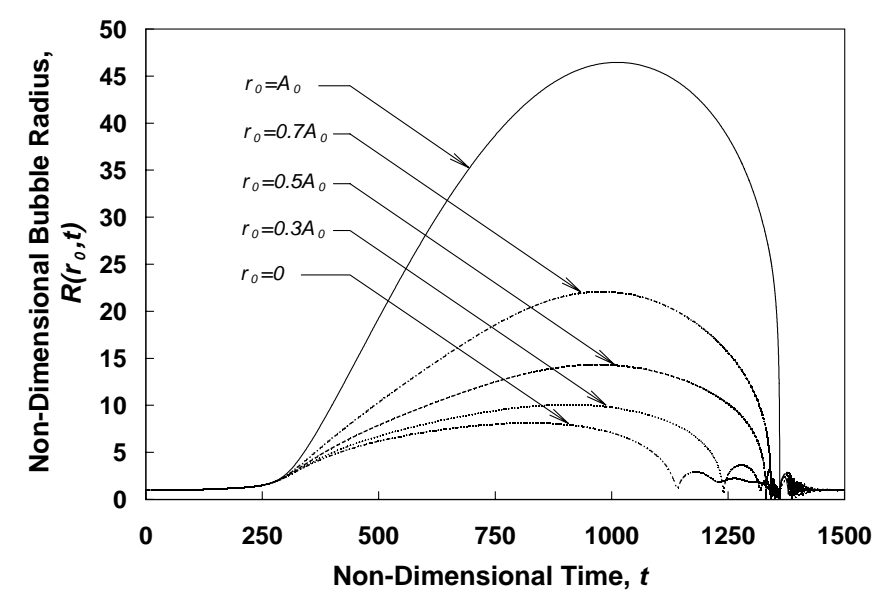

Figure 4: The time history of the dimensionless bubble size at five different positions in the cloud for $\alpha_{0}=0.02 \%$ and $D / A_{0}=10$ (corresponds to $t_{G}=1000$ ). Other parameters as in figure 2 . The cloud interaction parameter, $\beta \approx 2$.

spreading expansion wave causes all bubbles to grow and starts another cycle of cloud oscillation.

In the case of small $\beta$, the cloud exhibits very different dynamics, as shown in figure $4(\beta \approx 2)$. The bubbles in the cloud now have weaker interactions and, therefore, grow to a larger size. However, the inner bubbles still have a smaller growth rate than that of the outer bubbles. As a result, the inner bubbles grow to a size which is an order of magnitude smaller than that of the surface bubbles. But, more importantly, the inner bubbles collapse first. The collapse then spreads outward and results in an outward propagating shock, as shown in figure 5. Obviously, no geometric focusing occurs and the pressure and noise generated by the collapse are much smaller than when $\beta \gg 1$.

When $\beta$ has an intermediate order of magnitude, as illustrated in figure 6 , the collapse starts at mid-radius $\left(0.6 A_{0}\right.$ in the case of figure 6$)$ and spreads inward and outward from this location. The outward moving collapse tends to cancel the inward acceleration of the flow caused by the collapse of the bubbles on the cloud surface. The inward moving collapse has a structure similar to the shock wave described in figure 3 . However, the shock-enhancing effect is weaker than in the case of large $\beta$ due to the reduced "effective collapse size" of the cloud.

We note that Mørch, Hanson and Kedrinskii (Mørch, 1980, 1981, 1982; Hanson et al., 1981) first suggested that the collapse of a cloud of bubbles involves an inwardly propagating shock wave. Results of the present analysis confirm the formation of the shock waves which, however,

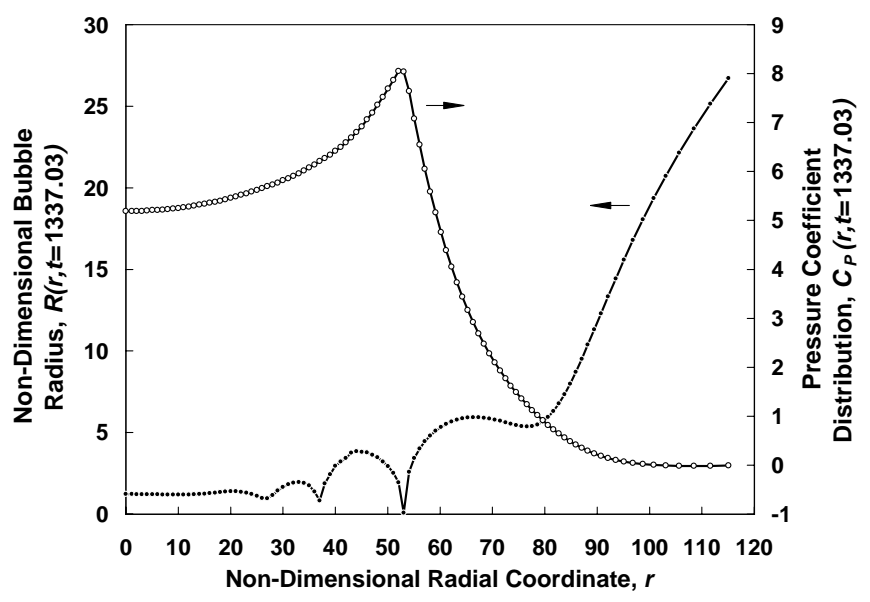

Figure 5: Bubble size and pressure distributions in the outward propagating shock wave at a sample moment in time, $t=1337.03$. Parameters as in figure 4 .

can propagate in either direction, depending on the value of $\beta$ in the cloud.

\subsection{Far-Field Noise Generated by the Cloud}

In this section, we examine the far-field noise generated by typical cloud dynamics. If $V^{*}\left(t^{*}\right)$ denotes the timevarying volume of the cloud, then the far-field acoustic pressure produced by the volumetric acceleration of the cloud is given by (Dowling and Ffowcs, 1983; Blake, 1986)

$$
p_{a}^{*}\left(t^{*}\right)=\frac{\rho_{L}^{*}}{4 \pi r^{*}} \frac{d^{2} V^{*}\left(t^{*}\right)}{d t^{*^{2}}},
$$

where $p_{a}^{*}$ is the dimensional radiated acoustic pressure and $r^{*}$ is the distance from the cloud center to the point of measurement. We have neglected the acoustic contributions from individual bubbles since they are minor in the far field. For present purposes a normalized far-field acoustic pressure is defined as

$$
\begin{aligned}
p_{a}(t) & =\frac{p_{a}^{*} r^{*}}{\frac{1}{2} \rho_{L}^{*} U^{*^{2}} D^{*}} \\
& =\frac{2 R_{0}}{D}\left[A^{2}(t) \frac{d^{2} A(t)}{d t^{2}}+2 A(t)\left(\frac{d A(t)}{d t}\right)^{2}\right]
\end{aligned}
$$

where the normalizing length-scale was chosen to be $D^{*}$, the typical length of the pressure perturbation experienced by the cloud. In practice, $D^{*}$ will be comparable to the size of the body, for example, the chord of a propeller blade. A typical example of the time history of the cloud radius and the far-field acoustic pressure is given in figure 7 . Here, the ratio of the pressure perturbation 


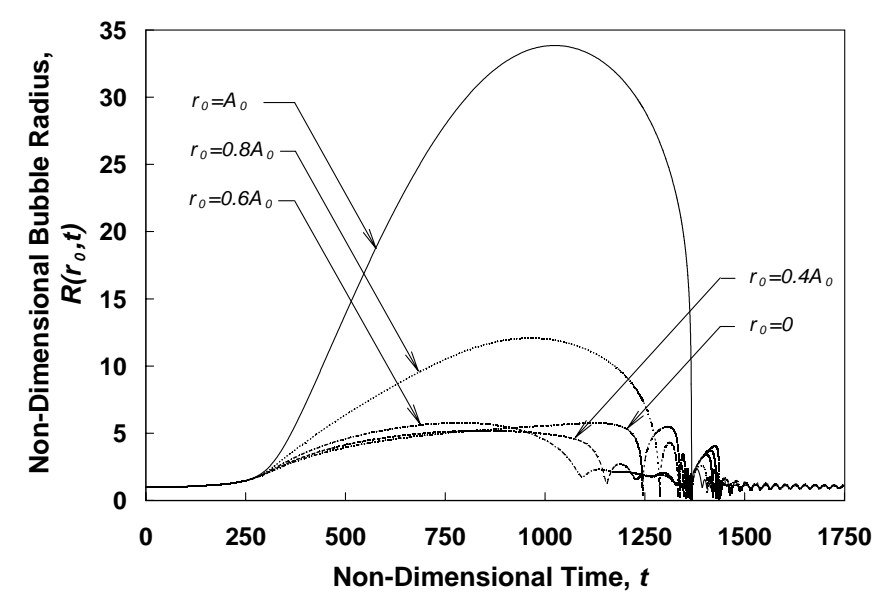

Figure 6: The time history of the dimensionless bubble size at five different positions in the cloud for $\alpha_{0}=0.1 \%$ and $D / A_{0}=10$ (corresponds to $t_{G}=1000$ ). Other parameters as in figure 2 . The cloud interaction parameter, $\beta \approx 10$.

length-scale to initial size of the cloud, $D / A_{0}$, is chosen as 2 (which corresponds to the duration of the pressure perturbation, $t_{G}=200$ ), small enough to cause the collapse to start from the cloud surface and, therefore, to result in coherent oscillation of the cloud. It is interesting that, unlike single bubbles, the cloud only collapses to a size marginally smaller than its equilibrium size. However, the void fraction within the cloud undergoes large changes. This is consistent with the recent experimental observations of unsteady cloud collapse by Reisman et al. (1997).

As illustrated in $\$ 4.2$, an inward propagating shock wave develops during the collapse process. When the enhanced shock wave reaches to the center of the cloud, large impulsive noise is generated, as shown in figure 7 . The first collapse is followed by successive rebounds and collapses which also produce radiated pulses. The magnitudes of the subsequent pulses decay with time due to the attenuation from bubble damping mechanisms. After several cycles, the cloud begins to oscillate at its natural frequency.

Figure 8 presents the power spectral density of the farfield noise in figure 7 as a function of dimensionless frequency, $f$, which is non-dimensionalized by the time scale, $R_{0}^{*} / U^{*}$. This spectrum exhibits an average $f^{-2}$ decay over the frequency range used. This behavior is typical of the cavitation noise associated with cavitating bodies or hydrofoils (see, for example, Blake et al., 1977; Arakeri and Shangumanathan, 1985; Ceccio and Brennen, 1991; Reisman et al., 1997). Other computational cases exhibited

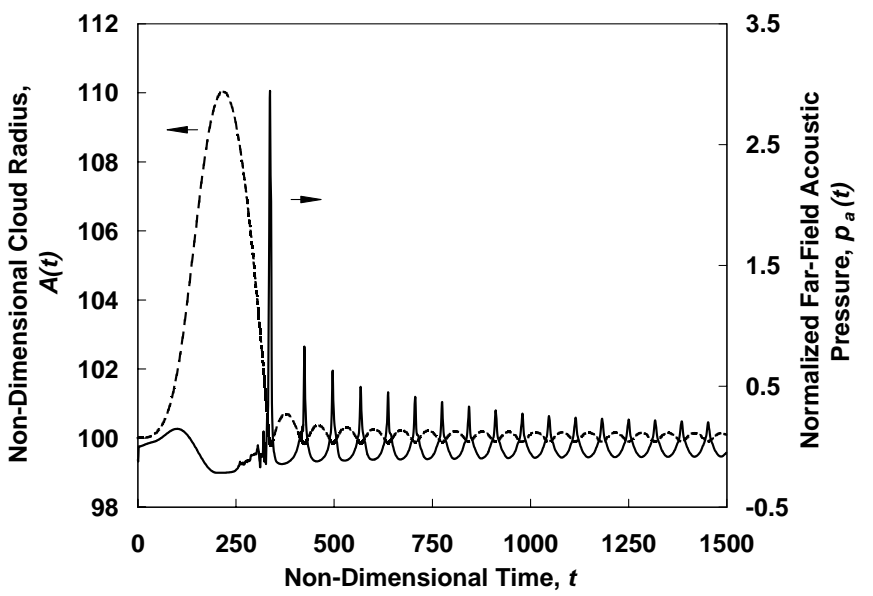

Figure 7: The time history of the dimensionless cloud radius and the resulting far-field noise for $\alpha_{0}=0.8 \%$ and $D / A_{0}=2$ (corresponds to $t_{G}=200$ ). Other parameters as in figure 2 .

an average $f^{-n}$ behavior with $n$ in the range of 0.5 to 2 .

The first and highest peak in figure 8 corresponds to the first natural frequency of the cloud, $f=0.015$ (or $1.5 \mathrm{kHz}$ in dimensional terms), in the present case. Higher harmonics of the first cloud natural frequency are also present in the spectrum and reflect the high degree of nonlinearity in the cloud dynamics. Another significant feature of the spectrum is that most of the energy is in the lower frequency range and is due to the coherent dynamics of the cloud. Note that in their cavitation experiments both Marboe et al. (1986) and Arakeri and Shangumanathan (1985) observed a tendency for the noise spectrum to shift towards lower frequencies at higher bubble densities. Bubble interactions were speculated to be the cause and this conjecture is strengthened by the present results.

To determine the magnitude of noise, the far-field acoustic impulse, $I$, is defined as the area under the largest pulse of the pressure signal or

$$
I=\int_{t_{1}}^{t_{2}} p_{a}(t) d t
$$

where $t_{1}$ and $t_{2}$ are times before and after the pulse at which the acoustic pressure, $p_{a}$, is zero. Figure 9 presents the acoustic impulse as a function of the cloud interaction parameter, $\beta$, for flows with different cavitation numbers, $\sigma$, and different ratios of the pressure perturbation lengthscale to initial cloud size, $D / A_{0}$. In all cases, the impulse increases with increasing $\beta$. Moreover, for larger $D / A_{0}$, there is a larger difference in different cavitation numbers. This is because the recovery of the ambient pressure of the cloud is slower and, therefore, the bubbles have more 


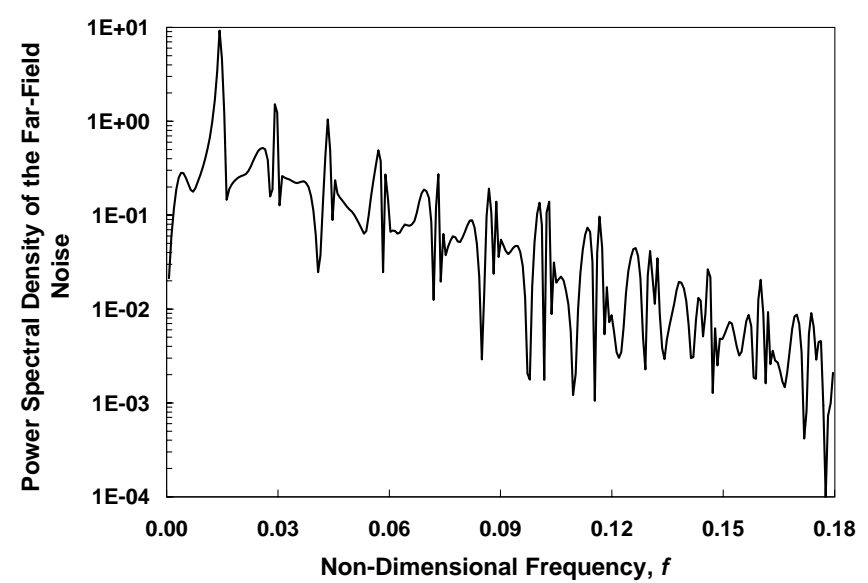

Figure 8: The non-dimensional power spectral density of the far-field noise in figure 7 as a function of dimensionless frequency. The lowest cloud natural frequency is about 0.015 . The natural frequency of single bubbles in the cloud is 0.158 .

time to cavitate. Under these circumstances the degree of cavitation in the cloud and the resulting acoustic impulse increases with decreasing cavitation number. On the other hand, if $D / A_{0}$ is small, the cloud will not have cavitated much before the recovery of the ambient pressure. Therefore, the influence of the cavitation number is smaller.

To investigate the relationship of the acoustic impulse to various parameters of the flow, calculations using a wide variety of parameter choices (45 permutations) were performed. It was found that the acoustic impulse is linearly correlated with the maximum total volume of the bubbles in the cloud normalized by the pressure perturbation length-scale, $V_{B M A X} /(0.5 D)^{3}$, as shown in figure 10. Moreover, as illustrated in figure 11 , this total volume decreases with increasing cavitation number and with increase in initial void fraction. It also varies with $D / A_{0}$.

\subsection{Shock speed}

Figure 12 shows the inward propagation speed of the spherical bubbly shock wave (normalized by $U^{*}$ ) as a function of the location of the shock front for three different initial void fractions, $\alpha_{0}$, of $0.03 \%, 0.3 \%$ and $3 \%$. In each case, the speed of the shock when it is initially formed in the surface layer of the cloud is of the order of $10 \mathrm{~m} / \mathrm{s}$. However, the speed increases as the strengthening shock propagates into the cloud. The increase in the speed is very pronounced over the last $20 \%$ of the collapse so that the shock speed near the center of the cloud is an order

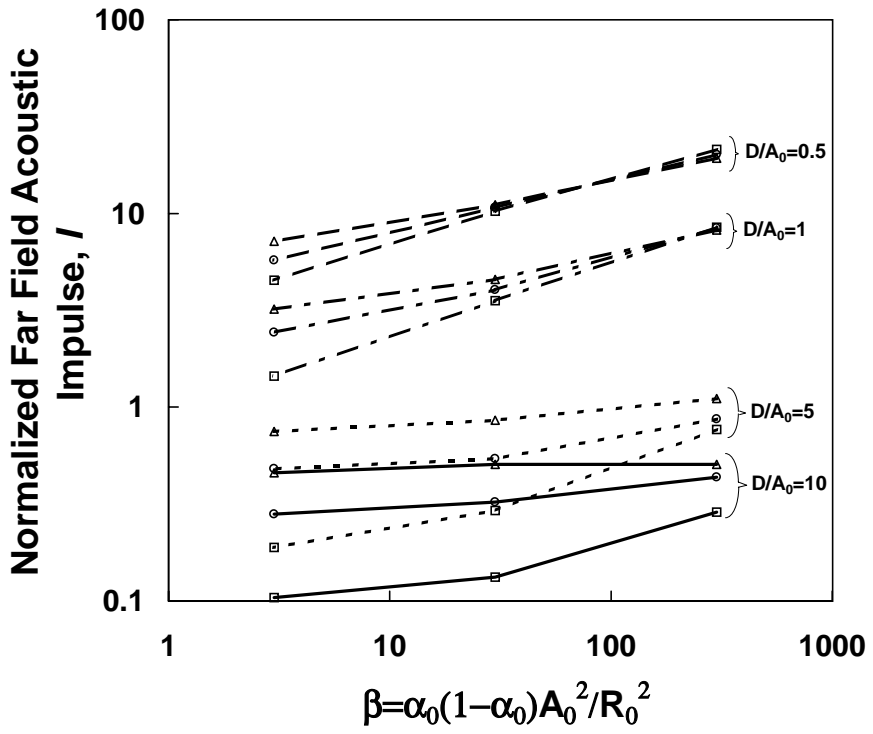

Figure 9: The impulse of the normalized far-field noise, $I$, as a function of the cloud interaction parameter, $\beta$, for different values of $D / A_{0}$ and three cavitation numbers, $\sigma$ $(0.45=\triangle, 0.55=\circ, 0.65=\square)$. Other parameters are $C_{P M I N}=-0.75$ and $A_{0} / R_{0}=100$.

of magnitude larger than that in the outer region. The speed is $O\left(10^{2} \mathrm{~m} / \mathrm{s}\right)$ for the larger void fractions used here and can reach $O\left(10^{4} \mathrm{~m} / \mathrm{s}\right)$ for the smallest void fraction $\left(\alpha_{0}=0.03 \%\right)$. If these shock pulses passed over a pressure transducer in a cavitating flow, the typical durations of the output signals would range from $10^{-4} \mathrm{sec}$ to $10^{-6} \mathrm{sec}$.

\subsection{Comparison with Experimental Observations}

Earlier measurements of the noise produced by cloud cavitation are characterized by pressure pulses of very short duration and large magnitude (see, for example, Bark and Berlekom, 1978; Shen and Peterson, 1978, 1980; Bark, 1985; Le et al., 1993; Reisman et al., 1994). However, the basic mechanism for the production of these pulses was not clear. The present theory suggests that the formation and concentration of the bubbly shock waves could be responsible. Recently, experimental investigations of the large unsteady and impulsive pressures which are experienced on the suction surface of an oscillating hydrofoil as a result of cloud cavitation were conducted (Brennen et al., 1996; Reisman et al., 1997). The experiments used piezo-electric transducers to measure the unsteady pressures at four locations along the chord of the foil and at two locations along the walls of the tunnel test section. The transducers on the foil surface registered very large positive pressure pulses with amplitudes of $O(10$ bar $)$ with 


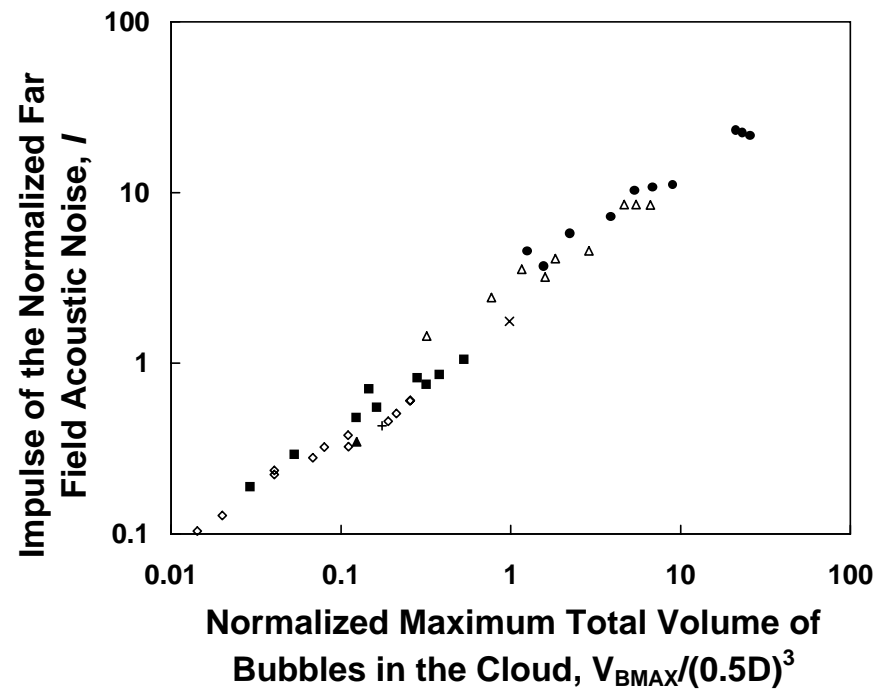

Figure 10: The impulse of the normalized far-field noise as a function of the normalized maximum total volume of bubbles in the cloud at different values of $D / A_{0}(31.25=$ solid $\triangle, 15.625=+, 10=\diamond, 5=$ solid $\square, 3.125=\times$, $1=\triangle, 0.5=\bullet)$. Three different cavitation numbers, $\sigma$, of $0.45,0.55$, and 0.65 , and three different initial void fractions, $\alpha_{0}$, of $0.03 \%, 0.3 \%$, and $3 \%$ are used. Other parameters are $C_{P M I N}=-0.75$ and three different initial cloud radii, $A_{0} / R_{0}$, of 31,100 , and 312 .

durations of $O\left(10^{-4} \mathrm{sec}\right)$. These orders of magnitudes are in the same range as the present calculations. Moreover, it was found that the pressure pulses moved with speeds of $O(10-100 \mathrm{~m} / \mathrm{sec})$ in agreement with the range predicted by the present theory.

\section{CONCLUDING REMARKS}

The nonlinear growth and collapse of a spherical cloud of cavitation bubbles has been computed using fully nonlinear continuum mixture equations coupled with the Rayleigh-Plesset equation for the dynamics of the bubbles. This system is solved numerically using a Lagrangian integral method. It is shown that a bubbly shock wave develops as part of the nonlinear collapse of the bubble cloud. The dynamics and acoustic consequences of the shock wave are strongly dependent on the cloud interaction parameter, $\beta=\alpha_{0}\left(1-\alpha_{0}\right) A_{0}^{2} / R_{0}^{2}$, where, $\alpha_{0}$ is the initial void fraction of the cloud, $A_{0}$ is the initial cloud radius and $R_{0}$ is the initial bubble radius. For a cloud with large $\beta$, the shock wave forms near the surface of the cloud and propagates inward. The strength and the speed of the shock increase considerably due to the geometric focusing. Very high pressure pulses are produced

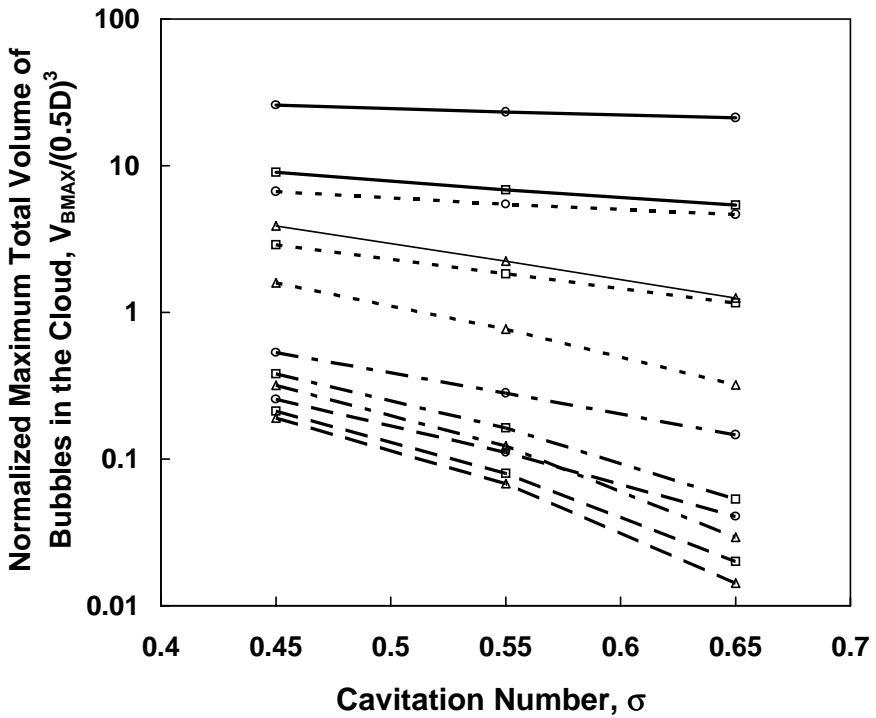

Figure 11: The normalized maximum total volume of bubbles in the cloud as a function of cavitation number. Data is shown for $C_{P M I N}=-0.75$, four different values of $D / A_{0}(0.5=$ solid lines, $1.0=$ dotted lines, $5.0=$ dash-dot lines, $10=$ dashed lines) and three initial void fractions, $\alpha_{0}(3 \%=\circ, 0.3 \%=\square, 0.03 \%=\triangle)$. Other parameters as in figure 4.

when the shock wave passes the bubbles in the cloud and causes them to collapse. Moreover, the enhanced shock produces very high pressures at the center of the cloud and then causes the rebound of the cloud. The volumetric acceleration of the cloud induces a large pulse in the far-field noise.

When $\beta$ is small, shock enhancement does not occur. Rather bubble collapse first occurs in the center of the cloud and results in an outward propagating shock wave. At intermediate $\beta$ the collapse starts at mid-radius and propagates inward and outward simultaneously. These shock wave dynamics, however, also depend on the duration of the pressure perturbation imposed on the cloud. If the cloud experiences a short period of depressurization, bubble collapse will start at the cloud surface.

Understanding such bubbly flow and shock wave processes is important because these flow structures propagate the noise and produce the impulsive loads on nearby solid surfaces in a cavitating flow. The results of this research suggest that large value of $\beta$ promotes the formation and focusing of a bubbly shock wave which is one of the major mechanisms for the enhanced noise and damage potential associated with cloud cavitation. Parenthetically, this requires either the initial void fraction or the ratio of cloud size to bubble size be sufficiently large and 


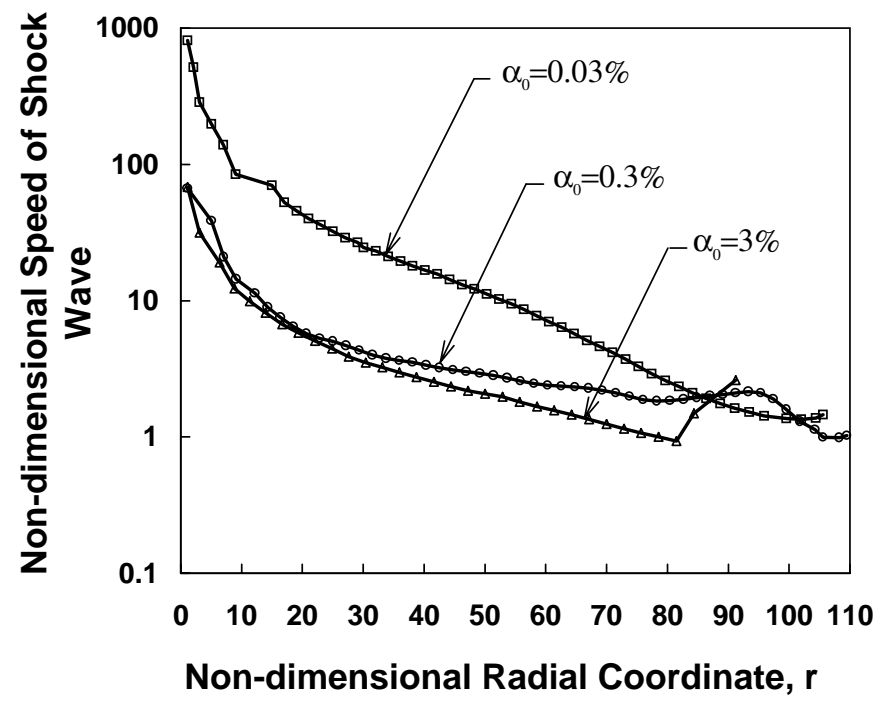

Figure 12: The speed of the inward propagating bubbly shock wave as a function of shock front position in the cloud for three different initial void fractions. Other parameters are $C_{P M I N}=-0.75, \sigma=0.45, D / A_{0}=5$, and $A_{0} / R_{0}=100$.

this, in turn, is qualitatively in accord with the observation that cavitation must be quite extensive for the cloud phenomenon to be manifest. Furthermore, this implies that bubble/bubble interaction effects play a crucial role in cloud cavitation noise and damage.

The theoretical results shed some light on previous experimental observations of cloud cavitation (Bark and Berlekom, 1978; Shen and Peterson, 1978, 1980; Bark, 1985; Franc and Michel, 1988; Kubota et al., 1989; Le et al., 1993; Reisman et al., 1994; Reisman and Brennen, 1996; Reisman et al., 1997). Experimental measurements of the noise produced by cloud cavitation all exhibit pressure pulses of very short duration and large amplitude. These pulses have magnitudes on the order of tens of atmospheres with typical durations of the order of tens of milliseconds (see, for example, Reisman et al., 1997). Moreover, these pulses appear to propagate through the bubbly cloud with speeds ranging from $O(10) \mathrm{m} / \mathrm{s}$ to $O(100) \mathrm{m} / \mathrm{s}$ (Brennen et al., 1996; Reisman et al., 1997). These magnitudes are consistent with the present calculations.

It also appears that an understanding of the collapse shock dynamics and acoustics has important consequences and implications for the scaling of cloud cavitation noise and damage. For example, the $\beta$ values could be very different for the model and the prototype; frequently, $\alpha_{0}$ and $R_{0}$ will be similar but $A_{0}$ will be quite different. This could cause severe cloud cavitation in the prototype which might not show up in the model if the model values of $\beta$ were small.

Finally we should note that, of course, most clouds are not spherical. Nevertheless the collapse of all or part of non-spherical clouds will produce points at which shock waves focus to produce large local and radiated pulses. Sturtevant and Kulkarny (1976) present a useful review of the various gasdynamic shock wave focusing phenomena including the effects of inhomogeneous media. However, it is not currently clear what three-dimensional forms the propagating bubbly shock waves might take in the highly non-uniform bubbly environments which occur in real cavitating flows.

\section{ACKNOWLEDGMENTS}

The authors are very grateful for the support for this research provided by the Office of Naval Research under Contract N00014-91-J-1295.

\section{REFERENCES}

Arakeri, V.H., and Shangumanathan, V., 1985, "On the Evidence for the Effect of Bubble Interference on Cavitation Noise," Journal of Fluid Mechanics, Vol. 159, pp. 131-150.

Bark, G., 1985, "Developments of Distortions in Sheet Cavitation on Hydrofoils," Proceedings of the ASME International Symposium on Jets and Cavities, pp. 470-493.

Bark, G., and Berlekom, W.B., 1978, "Experimental Investigations of Cavitation Noise," Proceedings of the 12th ONR Symposium on Naval Hydrodynamics, pp. 470-493.

Biesheuvel, A., and van Wijngaarden, L., 1984, "Two Phase Flow Equations for a Dilute Dispersion of Gas Bubbles in Liquid," Journal of Fluid Mechanics, Vol. 148, pp. $301-318$.

Birnir, B., and Smereka, P., 1990, "Existence Theory and Invariant Manifolds of Bubble Cloud," Communications on Pure and Applied Mathematics, Vol. 43, pp. 363413.

Blake, W.K., 1986, Mechanics of Flow-Induced Sound and Vibration, Academic Press.

Blake, W.K., Wolpert, M.J., and Geib, F.E., 1977, "Cavitation Noise and Inception As Influenced by Boundary-Layer Development on a Hydrofoil," Journal of Fluid Mechanics, Vol. 80, pp. 617-640.

Brennen, C.E., 1995, Cavitation and Bubble Dynamics, Oxford University Press, New York.

Brennen, C.E., Reisman, G.E., and Wang, Y.-C., 1996, "Shock Waves in Cloud Cavitation," Proceedings of the 
21th ONR Symposium on Naval Hydrodynamics, Trondheim, Norway.

Ceccio, S.L., and Brennen, C.E., 1991, "Observations of the Dynamics and Acoustics of Travelling Bubble Cavitation," Journal of Fluid Mechanics, Vol. 233, pp. 633-660.

Chahine, G.L., 1982a, "Pressure Field Generated by the Collective Collapse of Cavitation Bubbles," IAHR Symposium on Operating Problems of Pump Stations and Power Plants, Amsterdam, The Netherlands, Vol. 1(2), pp. 112.

Chahine, G.L., 1982b, "Cloud Cavitation: Theory," Proceedings of the 14th ONR Symposium on Naval Hydrodynamics, National Academy Press, pp. 165-194.

Chahine, G.L., and Duraiswami, R., 1992, "Dynamical Interactions in a Multibubble Cloud," ASME Journal of Fluids Engineering, Vol. 114, pp. 680-686.

Chahine, G.L., Duraiswami, R., and Rebut, M., 1992, "Analytical and Numerical Study of Large Bubble/Bubble and Bubble/Flow Interactions," Proceedings of the 19th ONR Symposium on Naval Hydrodynamics, Seoul, South Korea.

Chapman, R.B., and Plesset, M.S., 1971, "Thermal Effects in the Free Oscillation of Gas Bubbles," ASME Journal of Basic Engineering, Vol. 93, pp. 373-376.

d'Agostino, L., and Brennen, C.E., 1983, "On the Acoustical Dynamics of Bubble Clouds," ASME Cavitation and Multiphase Flow Forum, Houston, TX, pp. 7275 .

d'Agostino, L., and Brennen, C.E., 1988, "Acoustical Absorption and Scattering Cross-Sections of Spherical Bubble Clouds," Journal of the Acoustical Society of America, Vol. 84, pp. 2126-2134.

d'Agostino, L., and Brennen, C.E., 1989, "Linearized Dynamics of Spherical Bubble Clouds," Journal of Fluid Mechanics, Vol. 199, pp. 155-176.

Dowling, A.P., and Ffowcs Williams, J.E., 1983, Sound and Sources of Sound, Ellis Horwood Ltd. and John Wiley and Sons.

Franc, J.P., and Michel, J.M., 1988, "Unsteady Attached Cavitation on an Oscillating Hydrofoil," Journal of Fluid Mechanics, Vol. 193, pp. 171-189.

Hanson, I., Kedrinskii, V.K., and Mørch, K.A., 1981, "On the Dynamics of Cavity Clusters," Journal of Applied Physics, Vol. 15, pp. 1725-1734.

Ishii, M., 1975, Thermo-Fluid Dynamic Theory of TwoPhase Flow, Eyrolles.

Kameda, M., and Matsumoto, Y., 1995, "Structure of Shock Waves in a Liquid Containing Gas Bubbles,"
IUTAM Symposium on Waves in Liquid/Gas and Liquid/Vapour Two-Phase Systems, Morioka, S., and van Wijngaarden, L., ed., Kluwer Academic Publishers, Dordrecht, The Netherlands, pp. 117-126.

Knapp, R.T., Daily, J.W., and Hammitt, F.G., 1970, Cavitation, McGraw Hill, New York.

Kubota, A., Kato, H., Yamaguchi, H., and Maeda, M., 1989, "Unsteady Structure Measurement of Cloud Cavitation on a Foil Section Using Conditional Sampling," ASME Journal of Fluids Engineering, Vol. 111, pp. 204210.

Kubota, A., Kato, H., and Yamaguchi, H., 1992, "A New Modelling of Cavitating Flows: A Numerical Study of Unsteady Cavitation on a Hydrofoil Section," Journal of Fluid Mechanics, Vol. 240, pp. 59-96.

Kumar, S., and Brennen, C.E., 1991, "Non-linear Effects in the Dynamics of Clouds of Bubbles," Journal of the Acoustical Society of America, Vol. 89, pp. 707-714.

Kumar, S., and Brennen, C.E., 1992, "Harmonic Cascading in Bubble Clouds," Proceedings of the International Symposium on Propulsors and Cavitation, pp. 171179.

Kumar, S., and Brennen, C.E., 1993, "Some Nonlinear Interactive Effects in Bubbly Cavitating Clouds," Journal of Fluid Mechanics, Vol. 253, pp. 565-591.

Le, Q., Franc, J.M., and Michel, J.M., 1993, "Partial Cavities: Global Behaviour and Mean Pressure Distribution," ASME Journal of Fluids Engineering, Vol. 115, pp. 243-248.

Marboe, R.C., Billet, M.L., and Thompson, D.E., 1986, "Some Aspects of Traveling Bubble Cavitation and Noise," Proceedings of the ASME International Symposium on Cavitation and Multiphase Flow Noise, FED Vol. 45, pp. 119-126.

Mørch, K.A., 1980, "On the Collapse of Cavity Cluster in Flow Cavitation," Proceedings of the 1st International Conference on Cavitation and Inhomogenieties in Underwater Acoustics, Lauterborn, W., ed., Springer Series in Electrophysics, Vol. 4, pp. 95-100.

Mørch, K.A., 1981, "Cavity Cluster Dynamics and Cavitation Erosion," Proceedings of the ASME Cavitation and Polyphase Flow Forum, pp. 1-10.

Mørch, K.A., 1982, "Energy Considerations on the Collapse of Cavity Cluster," Applied Scientific Research, Vol. 38, p. 313.

Nigmatulin, R.I., 1991, Dynamics of Multiphase Media, Hemisphere, New York. 
Noordzij, L., 1973, "Shock Waves in Mixtures of Liquid and Air Bubbles," Ph.D. Thesis, Technishe Hogeschool, Twente, The Netherlands.

Noordzij, L., and van Wijngaarden, L., 1974, "Relaxation Effects, Caused by Relative Motion, on Shock Waves in Gas-Bubble/Liquid Mixtures," Journal of Fluid Mechanics, Vol. 66, pp. 115-143.

Omta, R., 1987, "Oscillations of a Cloud of Bubbles of Small and Not So Small Amplitude," Journal of the Acoustical Society of America, Vol. 82, pp. 1018-1033.

Plesset, M.S., and Prosperetti, A., 1977, "Bubble Dynamics and Cavitation," Annual Review of Fluid Mechanics, Vol. 9, pp. 145-185.

Prosperetti, A., 1988, "Bubble-Related Ambient Noise in the Ocean," Journal of the Acoustical Society of America, Vol. 84, pp. 1042-1054.

Reisman, G.E., and Brennen, C.E., 1996, "Pressure Pulses Generated by Cloud Cavitation," ASME Symposium on Cavitation and Gas-Liquid Flows in Fluid Machinery and Devices, San Diego, California, FED Vol. 236, pp. 319-328.

Reisman, G.E., McKenney, E.A., and Brennen, C.E., 1994, "Cloud Cavitation on an Oscillating Hydrofoil," Proceedings of the 20th ONR Symposium on Naval Hydrodynamics, pp. 78-89.

Reisman, G.E., Wang, Y.-C., and Brennen, C.E., 1997, "Observations of Shock Waves in Cloud Cavitation," (to appear in Journal of Fluid Mechanics).

Sangani, A.S., 1991, "A Pairwise Interaction Theory for Determining the Linear Acoustic Properties of Dilute Bubbly Liquids," Journal of Fluid Mechanics, Vol. 232, pp. 221-284.

Shen, Y., and Peterson, F.B., 1978, "Unsteady Cavitation on an Oscillating Hydrofoil," Proceedings of the 12th ONR Symposium on Naval Hydrodynamics, pp. 362-384.

Shen, Y., and Peterson, F.B., 1980, "The Influence of Hydrofoil Oscillation on Boundary Layer Transition and Cavitation Noise," Proceedings of the 13th ONR Symposium on Naval Hydrodynamics, pp. 221-241.

Smereka, P., and Banerjee, S., 1988, "The Dynamics of Periodically Driven Bubble Clouds," Physics of Fluids, Vol. 31(12), pp. 3519-3531.

Soyama, H., Kato, H., and Oba, R., 1992, "Cavitation Observations of Severely Erosive Vortex Cavitation Arising in a Centrifugal Pump," Proceedings of the Third IMechE International Conference on Cavitation, pp. 103110.
Sturtevant, B., Kulkarny, V.J., 1976, "The Focusing of Weak Shock Waves," Journal of Fluid Mechanics, Vol. 73, pp. 651-680.

Wade, R.B., and Acosta, A.J., 1966, "Experimental Observations on the Flow Past a Plano-Convex Hydrofoil," ASME Journal of Basic Engineering, Vol. 88, pp. 273283.

Wang, Y.-C., 1996, "Shock waves in bubbly cavitating flows," Ph.D. Thesis, California Institute of Technology, Pasadena, California.

Wang, Y.-C., and Brennen, C.E., 1994, "Shock Wave Development on the Collapse of a Cloud of Bubbles," ASME Cavitation and Multiphase Flow Forum, Furuya, O., and Katz, J., ed., FED Vol. 194, pp. 15-19.

Wang, Y.-C., and Brennen, C.E., 1995a, "Shock Wave and Noise in the Collapse of a Cloud of Cavitation Bubbles," Proceedings of the 20th International Symposium on Shock Waves, Sturtevant, S., Shepherd, J.E., and Hornung, H.G., ed., World Scientific, NJ, pp. 1213-1218.

Wang, Y.-C., and Brennen, C.E., 1995b, "The Noise Generated by the Collapse of a Cloud of Cavitation Bubbles," ASME/JSME Symposium on Cavitation and GasLiquid Flow in Fluid Machinery and Devices, Ceccio, S.L., Furukawa, A., and Kim, J.H., ed., FED Vol. 226, pp. 1729.

van Wijngaarden, L., 1964, "On the Collective Collapse of a Large Number of Gas Bubbles in Water," Proceedings of the 11th International Congress on Applied Mechanics, Springer-Verlag, Berlin, pp. 854-861. 